\title{
On a nonlocal integral boundary value problem of nonlinear Langevin equation with different fractional orders
}

\author{
Bashir Ahmad ${ }^{1 *}$ (D, Ahmed Alsaedi and Sara Salem ${ }^{1}$
}

\section{"Correspondence:}

bashirahmad_qau@yahoo.com 'Nonlinear Analysis and Applied Mathematics (NAAM)-Research

Group, Department of Mathematics, Faculty of Science, King Abdulaziz

University, Jeddah, Saudi Arabia

\section{Springer}

\begin{abstract}
In this paper we develop the existence theory for a nonlinear Langevin equation involving Caputo fractional derivatives of different orders and Riemann-Liouville fractional integral supplemented with nonlocal multi-point and multi-strip boundary conditions. We make use of the modern methods of functional analysis to obtain the existence and uniqueness results for the given problem, which are well illustrated with the aid of examples. Our results are new and correspond to some new ones for specific choices of the parameters involved in the problem.
\end{abstract}

\section{Introduction}

Langevin equation is an important equation of mathematical physics that is used in modeling the phenomena occurring in fluctuating environment such as Brownian motion. The classical form of this equation was derived in terms of ordinary derivatives by Paul Langevin in 1908 [1]. Langevin equation is also known as a stochastic differential equation as it governs the fast motion of microscopic variables (degrees of freedom) of the dynamical systems. However, the classical Langevin equation failed to describe the complex systems, and its several generalizations were proposed to model the physical phenomena in disordered regions [2], anomalous diffusion processes in complex and viscoelastic environment $[3,4]$, etc.

Fractional calculus, dealing with arbitrary order differential and integral operators, received great attention during the last few decades. Many researchers focused on developing the theoretical aspects, methods of solution, and applications of fractional differential equations. The popularity of the subject results from the nonlocal nature of fractional order operators, which can account for the past history of the phenomena under investigation. Now one can observe the shift in the study of mathematical models from integer order differential operators to their fractional order analogue. For application details in physics, biology, chemistry, medical sciences, etc., we refer the reader to the works [510].

There has been shown a great interest in the study of nonlocal nonlinear fractional order single-valued and multivalued boundary value problems in recent years. The interest in this topic results from the applicability of nonlocal and integral conditions in mathemati-

(c) The Author(s) 2019. This article is distributed under the terms of the Creative Commons Attribution 4.0 International License (http://creativecommons.org/licenses/by/4.0/), which permits unrestricted use, distribution, and reproduction in any medium, provided you give appropriate credit to the original author(s) and the source, provide a link to the Creative Commons license, and indicate if changes were made. 
cal modeling of many real world situations arising in applied and biological sciences. For details and examples, see [11-23] and the references cited therein.

Inspired by the wide application of tools of fractional calculus, the fractional analogue of Langevin equation was proposed by replacing the ordinary derivative by fractional order derivative in its classical form. Examples include motor single-file diffusion [24], KramersFokker-Planck equation and Langevin equation [25], control system [26], etc. Lim et al. [27] introduced a new form of Langevin equation containing two different fractional orders, which has made it possible to investigate fractal processes in a more flexible environment. For some recent works on fractional Langevin equation, see the papers [28-36] and the references cited therein.

In this paper, we consider the following nonlocal integral boundary value problem for a nonlinear Langevin equation with different order Caputo fractional derivatives:

$$
\begin{cases}{ }^{c} D^{\kappa}\left({ }^{c} D^{\zeta}+\chi\right) x(t)=f\left(t, x(t), I^{p} x(t)\right), & t \in[0,1], \\ x(0)=\sum_{j=1}^{m} v_{j} x\left(\sigma_{j}\right), \quad x^{\prime}(0)=0, & a_{1} x(1)+a_{2} x^{\prime}(1)=\sum_{i=1}^{n} \rho_{i} \int_{\xi_{i}}^{\eta_{i}} x(s) d s,\end{cases}
$$

where ${ }^{c} D^{\gamma}$ denotes the Caputo fractional derivative of order $\gamma(=\zeta, \kappa), p, \chi>0,0<\zeta \leq 1$, $1<\kappa \leq 2, a_{1}, a_{2}, v_{j}, \rho_{i} \in \mathbb{R}, 0<\sigma_{j}<\xi_{i}<\eta_{i}<1, i=1,2, \ldots, n, j=1,2, \ldots, m$, and $f:[0,1] \times$ $\mathbb{R} \times \mathbb{R} \rightarrow \mathbb{R}$ is a given continuous function.

We arrange the rest of the paper as follows. In Sect. 2, we present some definitions of fractional calculus and fixed point theorems related to our work. Section 3 contains the main results, while examples illustrating the main results are given in Sect. 4. Some interesting observations are presented in the concluding section.

\section{Preliminaries}

This section is devoted to some basic concepts of fractional calculus [6].

Definition 2.1 The Riemann-Liouville fractional integral $I_{a}^{\omega}$ of order $\omega \in \mathbb{R}(\omega>0)$ for a locally integrable real-valued function $u$ on the interval $(a, b)$ with $a, b \in \mathbb{R}$ is defined as

$$
I_{a}^{\omega} u(t)=\frac{1}{\Gamma(\omega)} \int_{a}^{t}(t-s)^{\omega-1} u(s) d s
$$

where $\Gamma$ denotes the Euler gamma function.

Definition 2.2 Let $u^{(m-1)} \in L^{1}[a, b]$ with $a, b \in \mathbb{R}$. The Riemann-Liouville fractional derivative $D_{a}^{\omega}$ of order $\omega(m-1<\omega<m, m \in \mathbb{N})$ for $u$ is defined as

$$
D_{a}^{\omega} u(t)=\frac{d^{m}}{d t^{m}} I_{a}^{1-\omega} u(t)=\frac{1}{\Gamma(m-\omega)} \frac{d^{m}}{d t^{m}} \int_{a}^{t}(t-s)^{m-1-\omega} u(s) d s
$$

The Caputo fractional derivative ${ }^{c} D_{a}^{\omega}$ of order $\omega(m-1<\omega<m, m \in \mathbb{N})$ is defined by

$$
{ }^{c} D_{a}^{\omega} u(t)=D_{a}^{\omega}\left[u(t)-u(a)-u^{\prime}(a) \frac{(t-a)}{1 !}-\cdots-u^{(m-1)}(a) \frac{(t-a)^{m-1}}{(m-1) !}\right] .
$$


Remark 2.3 If $u \in C^{m}[a, b]$, then the Caputo fractional derivative ${ }^{c} D_{a}^{\omega}$ of order $\omega(m-1<$ $\omega<m, m \in \mathbb{N}$ ) is defined as

$$
{ }^{c} D_{a}^{\omega}[u](t)=I_{a}^{1-\omega} u^{(m)}(t)=\frac{1}{\Gamma(m-\omega)} \int_{a}^{t}(t-s)^{m-1-\omega} u^{(m)}(s) d s .
$$

In the present work, we denote the Riemann-Liouville fractional integral $I_{a}^{\omega}$ and the Caputo fractional derivative ${ }^{c} D_{a}^{\omega}$ with $a=0$ by $I^{\omega}$ and ${ }^{c} D^{\omega}$, respectively.

\section{Main results}

Before presenting the main results, we prove an auxiliary lemma related to the linear variant of problem (1.1). This lemma plays an important role in transforming the given problem to a fixed point problem.

\subsection{Auxiliary lemma}

Lemma 3.1 Let $g \in C([0,1], \mathbb{R})$. Then the problem consisting of linear fractional differential equation

$$
{ }^{c} D^{\kappa}\left({ }^{c} D^{\zeta}+\chi\right) x(t)=g(t), \quad 1<\kappa \leq 2,0<\zeta<1,
$$

and the boundary conditions of (1.1) is equivalent to the integral equation

$$
\begin{aligned}
x(t)= & \int_{0}^{t} \frac{(t-s)^{\zeta+\kappa-1}}{\Gamma(\zeta+\kappa)} g(s) d s-\chi \int_{0}^{t} \frac{(t-s)^{\zeta-1}}{\Gamma(\zeta)} x(s) d s \\
& -\mu_{1}(t) \sum_{j=1}^{m} v_{j} \int_{0}^{\sigma_{j}}\left(\frac{\left(\sigma_{j}-s\right)^{\zeta+\kappa-1}}{\Gamma(\zeta+\kappa)} g(s)-\chi \frac{\left(\sigma_{j}-s\right)^{\zeta-1}}{\Gamma(\zeta)} x(s)\right) d s \\
& +\mu_{2}(t)\left[\sum_{i=1}^{n} \rho_{i} \int_{\xi_{i}}^{\eta_{i}} \int_{0}^{s}\left(\frac{(s-u)^{\zeta+\kappa-1}}{\Gamma(\zeta+\kappa)} g(u)-\chi \frac{(s-u)^{\zeta-1}}{\Gamma(\zeta)} x(u)\right) d u d s\right. \\
& -a_{1} \int_{0}^{1}\left(\frac{(1-s)^{\zeta+\kappa-1}}{\Gamma(\zeta+\kappa)} g(s)-\chi \frac{(1-s)^{\zeta-1}}{\Gamma(\zeta)} x(s)\right) d s \\
& \left.-a_{2} \int_{0}^{1}\left(\frac{(1-s)^{\zeta+\kappa-2}}{\Gamma(\zeta+\kappa-1)^{2}} g(s)-\chi \frac{(1-s)^{\zeta-2}}{\Gamma(\zeta-1)} x(s)\right) d s\right]
\end{aligned}
$$

where

$$
\begin{aligned}
& \mu_{1}(t)=\frac{1}{\Delta}\left(B_{1}-B_{2}\left(\frac{t^{\zeta+1}}{\Gamma(\zeta+2)}\right)\right), \quad \mu_{2}(t)=\frac{1}{\Delta}\left(A_{1}-A_{2}\left(\frac{t^{\zeta+1}}{\Gamma(\zeta+2)}\right)\right), \\
& \Delta=A_{1} B_{2}-A_{2} B_{1} \neq 0, \\
& A_{1}=-\sum_{j=1}^{m} v_{j} \frac{\sigma_{j}^{\zeta+1}}{\Gamma(\zeta+2)}, \quad A_{2}=1-\sum_{j=1}^{m} v_{j}, \\
& B_{1}=\frac{a_{1}+(\zeta+1) a_{2}}{\Gamma(\zeta+2)}-\sum_{i=1}^{n} \rho_{i} \frac{\eta_{i}^{\zeta+2}-\xi_{i}^{\zeta+2}}{\Gamma(\zeta+3)}, \quad B_{2}=a_{1}-\sum_{i=1}^{n} \rho_{i}\left(\eta_{i}-\xi_{i}\right) .
\end{aligned}
$$


Proof Applying the integral operator $I^{\kappa}$ to both sides of (3.1) and then the operator $I^{\zeta}$ to the resulting equation, we get

$$
\begin{aligned}
& x(t)=I^{\zeta+\kappa} g(t)-\chi I^{\zeta} x(t)+c_{0} \frac{t^{\zeta}}{\Gamma(\zeta+1)}+c_{1} \frac{t^{\zeta+1}}{\Gamma(\zeta+2)}+c_{2}, \\
& x^{\prime}(t)=I^{\zeta+\kappa-1} g(t)-\chi I^{\zeta-1} x(t)+c_{0} \frac{t^{\zeta-1}}{\Gamma(\zeta)}+c_{1} \frac{t^{\zeta}}{\Gamma(\zeta+1)},
\end{aligned}
$$

where $c_{0}, c_{1}, c_{2}$ are arbitrary constants.

Using the condition $x^{\prime}(0)=0$ in (3.8), we find that $c_{0}=0$. Inserting the value of $c_{0}$ in (3.7) and making use of the condition $x(0)=\sum_{j=1}^{m} v_{j} x\left(\sigma_{j}\right)$ together with the notations (3.5), we get

$$
A_{1} c_{1}+A_{2} c_{2}=J_{1}
$$

where

$$
J_{1}=\sum_{j=1}^{m} v_{j}\left(I^{\zeta+\kappa} g\left(\sigma_{j}\right)-\chi I^{\zeta} x\left(\sigma_{j}\right)\right) .
$$

Combining the condition $a_{1} x(1)+a_{2} x^{\prime}(1)=\sum_{i=1}^{n-2} \rho_{i} \int_{\xi_{i}}^{\eta_{i}} x(s) d s$ with (3.7) and (3.8), and using the notations (3.6) yields

$$
B_{1} c_{1}+B_{2} c_{2}=J_{2}
$$

where

$$
\begin{aligned}
J_{2}= & \sum_{i=1}^{n} \rho_{i} \int_{\xi_{i}}^{\eta_{i}}\left(I^{\zeta+\kappa} g(s)-\chi I^{\zeta} x(s)\right) d s-a_{1}\left(I^{\zeta+\kappa} g(1)-\chi I^{\zeta} x(1)\right) \\
& -a_{2}\left(I^{\zeta+\kappa-1} g(1)-\chi I^{\zeta-1} x(1)\right) .
\end{aligned}
$$

Solving systems (3.9) and (3.11) for $c_{1}$ and $c_{2}$, we find that

$$
c_{1}=\frac{B_{2} J_{1}-A_{2} J_{2}}{\Delta}, \quad c_{2}=\frac{A_{1} J_{2}-B_{1} J_{1}}{\Delta},
$$

where $\Delta$ is given by (3.4). Substituting the values of $c_{0}, c_{1}$, and $c_{2}$ in (3.7) together with the notations (3.3), we obtain solution (3.2). By direct computation, one can obtain the converse of the lemma. This completes the proof.

Denote by $X$ the Banach space of all continuous functions from $[0,1] \rightarrow \mathbb{R}$ endowed with norm $\|x\|=\sup \{|x(t)|: t \in[0,1]\}$. By Lemma 3.1, we transform problem (1.1) into a fixed point problem as $x=\mathcal{A} x$, where the operator $\mathcal{A}: X \rightarrow X$ is defined by

$$
\begin{aligned}
(\mathcal{A} x)(t)= & \int_{0}^{t} \frac{(t-s)^{\zeta+\kappa-1}}{\Gamma(\zeta+\kappa)} f\left(s, x(s), I^{p} x(s)\right) d s-\chi \int_{0}^{t} \frac{(t-s)^{\zeta-1}}{\Gamma(\zeta)} x(s) d s \\
& -\mu_{1}(t) \sum_{j=1}^{m} v_{j} \int_{0}^{\sigma_{j}}\left(\frac{\left(\sigma_{j}-s\right)^{\zeta+\kappa-1}}{\Gamma(\zeta+\kappa)} f\left(s, x(s), I^{p} x(s)\right)-\chi \frac{\left(\sigma_{j}-s\right)^{\zeta-1}}{\Gamma(\zeta)} x(s)\right) d s
\end{aligned}
$$




$$
\begin{aligned}
& +\mu_{2}(t)\left[\sum _ { i = 1 } ^ { n } \rho _ { i } \int _ { \xi _ { i } } ^ { \eta _ { i } } \int _ { 0 } ^ { s } \left(\frac{(s-u)^{\zeta+\kappa-1}}{\Gamma(\zeta+\kappa)} f\left(u, x(u), I^{p} x(u)\right)\right.\right. \\
& \left.-\chi \frac{(s-u)^{\zeta-1}}{\Gamma(\zeta)} x(u)\right) d u d s \\
& -a_{1} \int_{0}^{1}\left(\frac{(1-s)^{\zeta+\kappa-1}}{\Gamma(\zeta+\kappa)} f\left(s, x(s), I^{p} x(s)\right)-\chi \frac{(1-s)^{\zeta-1}}{\Gamma(\zeta)} x(s)\right) d s \\
& \left.-a_{2} \int_{0}^{1}\left(\frac{(1-s)^{\zeta+\kappa-2}}{\Gamma(\zeta+\kappa-1)} f\left(s, x(s), I^{p} x(s)\right)-\chi \frac{(1-s)^{\zeta-2}}{\Gamma(\zeta-1)} x(s)\right) d s\right] \\
& t \in[0,1]
\end{aligned}
$$

For the sake of computational convenience, we set

$$
\begin{aligned}
\Lambda_{1}= & \frac{1}{\Gamma(\zeta+\kappa+1)}+\bar{\mu}_{1} \sum_{j=1}^{m}\left|v_{j}\right| \frac{\sigma_{j}^{\zeta+\kappa}}{\Gamma(\zeta+\kappa+1)} \\
& +\bar{\mu}_{2}\left(\sum_{i=1}^{n}\left|\rho_{i}\right| \frac{\left(\eta_{i}^{\zeta+\kappa+1}-\xi_{i}^{\zeta+\kappa+1}\right)}{\Gamma(\zeta+\kappa+2)}+\frac{\left|a_{1}\right|}{\Gamma(\zeta+\kappa+1)}+\frac{\left|a_{2}\right|}{\Gamma(\zeta+\kappa)}\right) \\
\Lambda_{2}= & \chi\left[\frac{1}{\Gamma(\zeta+1)}+\bar{\mu}_{1} \sum_{j=1}^{m}\left|v_{j}\right| \frac{\sigma_{j}^{\zeta}}{\Gamma(\zeta+1)}\right. \\
& \left.+\bar{\mu}_{2}\left(\sum_{i=1}^{n}\left|\rho_{i}\right| \frac{\left(\eta_{i}^{\zeta+1}-\xi_{i}^{\zeta+1}\right)}{\Gamma(\zeta+2)}+\frac{\left|a_{1}\right|}{\Gamma(\zeta+1)}+\frac{\left|a_{2}\right|}{\Gamma(\zeta)}\right)\right]
\end{aligned}
$$

where

$$
\begin{aligned}
& \bar{\mu}_{1}=\max _{t \in[0,1]}\left|\mu_{1}(t)\right|=\frac{1}{|\triangle|}\left(\left|B_{1}\right|+\frac{\left|B_{2}\right|}{\Gamma(\zeta+2)}\right), \\
& \bar{\mu}_{2}=\max _{t \in[0,1]}\left|\mu_{2}(t)\right|=\frac{1}{|\triangle|}\left(\left|A_{1}\right|+\frac{\left|A_{2}\right|}{\Gamma(\zeta+2)}\right) .
\end{aligned}
$$

\subsection{Existence results}

In this subsection we prove the existence results for the given problem; the first one relies on Krasnoselskii's fixed point theorem [37], while the second one is based on the LeraySchauder nonlinear alternative [38].

Theorem 3.2 Let $f:[0,1] \times \mathbb{R}^{2} \rightarrow \mathbb{R}$ be a continuous function satisfying the conditions:

$\left(C_{1}\right)\left|f(t, x, y)-f\left(t, x_{1}, y_{1}\right)\right| \leq l\left(\left|x-x_{1}\right|+\left|y-y_{1}\right|\right)$ for all $t \in[0,1], l>0, x, y, x_{1}, y_{1} \in \mathbb{R}$;

$\left(C_{2}\right)|f(t, x, y)| \leq k(t)$ for all $(t, x, y) \in[0,1] \times \mathbb{R}^{2}$ and $k \in C\left([0,1], \mathbb{R}^{+}\right)$.

Then the boundary value problem (1.1) has at least one solution on $[0,1]$ if

$$
\Lambda_{3}<1
$$

where $\Lambda_{3}=l\left(1+\frac{1}{\Gamma(p+1)}\right)\left(\Lambda_{1}-\frac{1}{\Gamma(\zeta+\kappa+1)}\right)+\Lambda_{2}-\frac{\chi}{\Gamma(\zeta+1)}, \Lambda_{1}$ and $\Lambda_{2}$ are respectively given by (3.14) and (3.15) with $\Lambda_{2}<1$. 
Proof By assumption $\left(C_{2}\right)$, we fix $r \geq \Lambda_{1}\|k\| /\left(1-\Lambda_{2}\right)$ and define a closed ball $B_{r}=\{x \in X$ : $\|x\| \leq r\}$. Introduce the operators $\mathcal{A}_{1}$ and $\mathcal{A}_{2}$ on $B_{r}$ as

$$
\begin{aligned}
\left(\mathcal{A}_{1} x\right)(t)= & \int_{0}^{t} \frac{(t-s)^{\zeta+\kappa-1}}{\Gamma(\zeta+\kappa)} f\left(s, x(s), I^{p} x(s)\right) d s-\chi \int_{0}^{t} \frac{(t-s)^{\zeta-1}}{\Gamma(\zeta)} x(s) d s \\
\left(\mathcal{A}_{2} x\right)(t)= & -\mu_{1}(t) \sum_{j=1}^{m} v_{j} \int_{0}^{\sigma_{j}}\left(\frac{\left(\sigma_{j}-s\right)^{\zeta+\kappa-1}}{\Gamma(\zeta+\kappa)} f\left(s, x(s), I^{p} x(s)\right)-\chi \frac{\left(\sigma_{j}-s\right)^{\zeta-1}}{\Gamma(\zeta)} x(s)\right) d s \\
& +\mu_{2}(t)\left[\sum _ { i = 1 } ^ { n } \rho _ { i } \int _ { \xi _ { i } } ^ { \eta _ { i } } \int _ { 0 } ^ { s } \left(\frac{(s-u)^{\zeta+\kappa-1}}{\Gamma(\zeta+\kappa)} f\left(u, x(u), I^{p} x(u)\right)\right.\right. \\
& \left.-\chi \frac{(s-u)^{\zeta-1}}{\Gamma(\zeta)} x(u)\right) d u d s \\
& -a_{1} \int_{0}^{1}\left(\frac{(1-s)^{\zeta+\kappa-1}}{\Gamma(\zeta+\kappa)} f\left(s, x(s), I^{p} x(s)\right)-\chi \frac{(1-s)^{\zeta-1}}{\Gamma(\zeta)} x(s)\right) d s \\
& \left.-a_{2} \int_{0}^{1}\left(\frac{(1-s)^{\zeta+\kappa-2}}{\Gamma(\zeta+\kappa-1)} f\left(s, x(s), I^{p} x(s)\right)-\chi \frac{(1-s)^{\zeta-2}}{\Gamma(\zeta-1)} x(s)\right) d s\right]
\end{aligned}
$$

$t \in[0,1]$. Observe that $\mathcal{A} x=\mathcal{A}_{1} x+\mathcal{A}_{2} x, x \in B_{r}$ on $[0,1]$. In order to apply the conclusion of Krasnoselskii's fixed point theorem [37], we verify its hypothesis in three steps.

(i) For $x, y \in B_{r}$, we show that $\mathcal{A}_{1} x+\mathcal{A}_{2} y \in B_{r}$. For that, we have

$$
\begin{aligned}
\| \mathcal{A}_{1} x & +\mathcal{A}_{2} y \| \\
= & \sup _{t \in[0,1]}\left|\mathcal{A}_{1} x(t)+\mathcal{A}_{2} y(t)\right| \\
\leq & \sup _{t \in[0,1]}\left\{\int_{0}^{t} \frac{(t-s)^{\zeta+\kappa-1}}{\Gamma(\zeta+\kappa)}\left|f\left(s, x(s), I^{p} x(s)\right)\right| d s+\chi \int_{0}^{t} \frac{(t-s)^{\zeta-1}}{\Gamma(\zeta)}|x(s)| d s\right. \\
& +\left|\mu_{1}(t)\right| \sum_{j=1}^{m}\left|v_{j}\right| \int_{0}^{\sigma_{j}}\left(\frac{\left(\sigma_{j}-s\right)^{\zeta+\kappa-1}}{\Gamma(\zeta+\kappa)}\left|f\left(s, x(s), I^{p} x(s)\right)\right|+\chi \frac{\left(\sigma_{j}-s\right)^{\zeta-1}}{\Gamma(\zeta)}|x(s)|\right) d s \\
& +\left|\mu_{2}(t)\right|\left[\sum _ { i = 1 } ^ { n } | \rho _ { i } | \int _ { \xi _ { i } } ^ { \eta _ { i } } \int _ { 0 } ^ { s } \left(\frac{(s-u)^{\zeta+\kappa-1}}{\Gamma(\zeta+\kappa)}\left|f\left(u, x(u), I^{p} x(u)\right)\right|\right.\right. \\
& \left.+\chi \frac{(s-u)^{\zeta-1}}{\Gamma(\zeta)}|x(u)|\right)^{\prime} d u d s \\
& +\left|a_{1}\right|\left[\int_{0}^{1} \frac{(1-s)^{\zeta+\kappa-1}}{\Gamma(\zeta+\kappa)}\left|f\left(s, x(s), I^{p} x(s)\right)\right| d s+\chi \int_{0}^{1} \frac{(1-s)^{\zeta-1}}{\Gamma(\zeta)}|x(s)| d s\right] \\
& \left.\left.+\left|a_{2}\right|\left[\int_{0}^{1} \frac{(1-s)^{\zeta+\kappa-2}}{\Gamma(\zeta+\kappa-1)}\left|f\left(s, x(s), I^{p} x(s)\right)\right| d s+\chi \int_{0}^{1} \frac{(1-s)^{\zeta-2}}{\Gamma(\zeta-1)}|x(s)| d s\right]\right]\right\} \\
\leq & \|k\| \sup _{t \in[0,1]}\left\{\int_{0}^{t} \frac{(t-s)^{\zeta+\kappa-1}}{\Gamma(\zeta+\kappa)} d s+\left|\mu_{1}(t)\right| \sum_{j=1}^{m}\left|v_{j}\right| \int_{0}^{\sigma_{j}} \frac{\left(\sigma_{j}-s\right)^{\zeta+\kappa-1}}{\Gamma(\zeta+\kappa)} d s\right. \\
& +\left|\mu_{2}(t)\right|\left[\sum_{i=1}^{n}\left|\rho_{i}\right| \int_{\xi_{i}}^{\eta_{i}} \int_{0}^{s} \frac{(s-u)^{\zeta+\kappa-1}}{\Gamma(\zeta+\kappa)} d u d s+\left|a_{1}\right| \int_{0}^{1} \frac{(1-s)^{\zeta+\kappa-1}}{\Gamma(\zeta+\kappa)} d s\right. \\
& \left.\left.+\left|a_{2}\right| \int_{0}^{1} \frac{(1-s)^{\zeta+\kappa-2}}{\Gamma(\zeta+\kappa-1)} d s\right]\right\}
\end{aligned}
$$




$$
\begin{aligned}
+ & +\chi\|x\| \sup _{t \in[0,1]}\left\{\int_{0}^{t} \frac{(t-s)^{\zeta-1}}{\Gamma(\zeta)} d s+\left|\mu_{1}(t)\right| \sum_{j=1}^{m}\left|v_{j}\right| \int_{0}^{\sigma_{j}} \frac{\left(\sigma_{j}-s\right)^{\zeta-1}}{\Gamma(\zeta)} d s\right. \\
+ & \left|\mu_{2}(t)\right|\left[\sum_{i=1}^{n}\left|\rho_{i}\right| \int_{\xi_{i}}^{\eta_{i}} \int_{0}^{s} \frac{(s-u)^{\zeta-1}}{\Gamma(\zeta)} d u d s+\left|a_{1}\right| \int_{0}^{1} \frac{(1-s)^{\zeta-1}}{\Gamma(\zeta)} d s\right. \\
& \left.\left.+\left|a_{2}\right| \int_{0}^{1} \frac{(1-s)^{\zeta-2}}{\Gamma(\zeta-1)} d s\right]\right\} \\
\leq & \|k\|\left\{\frac{1}{\Gamma(\zeta+\kappa+1)}+\bar{\mu}_{1} \sum_{j=1}^{m}\left|v_{j}\right| \frac{\sigma_{j}^{\zeta+\kappa}}{\Gamma(\zeta+\kappa+1)}\right. \\
& \left.+\bar{\mu}_{2}\left(\sum_{i=1}^{n}\left|\rho_{i}\right| \frac{\left(\eta_{i}^{\zeta+\kappa+1}-\xi_{i}^{\zeta+\kappa+1}\right)}{\Gamma(\zeta+\kappa+2)}+\frac{\left|a_{1}\right|}{\Gamma(\zeta+\kappa+1)}+\frac{\left|a_{2}\right|}{\Gamma(\zeta+\kappa)}\right)\right\} \\
& +\chi\|x\|\left\{\frac{1}{\Gamma(\zeta+1)}+\bar{\mu}_{1} \sum_{j=1}^{m}\left|v_{j}\right| \frac{\sigma_{j}^{\zeta}}{\Gamma(\zeta+1)}\right. \\
& \left.+\bar{\mu}_{2}\left(\sum_{i=1}^{n}\left|\rho_{i}\right| \frac{\left(\eta_{i}^{\zeta+1}-\xi_{i}^{\zeta+1}\right)}{\Gamma(\zeta+2)}+\frac{\left|a_{1}\right|}{\Gamma(\zeta+1)}+\frac{\left|a_{2}\right|}{\Gamma(\zeta)}\right)\right\} \\
\leq & \|k\| \Lambda_{1}+\Lambda_{2} r \leq r,
\end{aligned}
$$

which shows that $\mathcal{A}_{1} x+\mathcal{A}_{2} y \in B_{r}$.

(ii) We establish that $\mathcal{A}_{2}$ is a contraction. For $x, y \in X$ and for each $t \in[0,1]$, we obtain

$$
\begin{aligned}
\| \mathcal{A}_{2} x & -\mathcal{A}_{2} y \| \\
\leq & \sup _{t \in[0,1]}\left\{| \mu _ { 1 } ( t ) | \sum _ { j = 1 } ^ { m } | v _ { j } | \int _ { 0 } ^ { \sigma _ { j } } \left(\frac{\left(\sigma_{j}-s\right)^{\zeta+\kappa-1}}{\Gamma(\zeta+\kappa)}\left|f\left(s, x(s), I^{p} x(s)\right)-f\left(s, y(s), I^{p} y(s)\right)\right|\right.\right. \\
& \left.+\chi \frac{\left(\sigma_{j}-s\right)^{\zeta-1}}{\Gamma(\zeta)}|x(s)-y(s)|\right) d s \\
& +\left|\mu_{2}(t)\right|\left[\sum _ { i = 1 } ^ { n } | \rho _ { i } | \int _ { \xi _ { i } } ^ { \eta _ { i } } \int _ { 0 } ^ { s } \left(\frac{(s-u)^{\zeta+\kappa-1}}{\Gamma(\zeta+\kappa)}\left|f\left(u, x(u), I^{p} x(u)\right)-f\left(u, y(u), I^{p} y(u)\right)\right|\right.\right. \\
& \left.+\chi \frac{(s-u)^{\zeta-1}}{\Gamma(\zeta)}|x(u)-y(u)|\right) d u d s \\
& +\left|a_{1}\right|\left[\int_{0}^{1} \frac{(1-s)^{\zeta+\kappa-1}}{\Gamma(\zeta+\kappa)}\left|f\left(s, x(s), I^{p} x(s)\right)-f\left(s, y(s), I^{p} y(s)\right)\right| d s\right. \\
& \left.+\chi \int_{0}^{1} \frac{(1-s)^{\zeta-1}}{\Gamma(\zeta)}|x(s)-y(s)| d s\right] \\
& +\left|a_{2}\right|\left[\int_{0}^{1} \frac{(1-s)^{\zeta+\kappa-2}}{\Gamma(\zeta+\kappa-1)}\left|f\left(s, x(s), I^{p} x(s)\right)-f\left(s, y(s), I^{p} y(s)\right)\right| d s\right. \\
& \left.\left.\left.+\chi \int_{0}^{1} \frac{(1-s)^{\zeta-2}}{\Gamma(\zeta-1)}|x(s)-y(s)| d s\right]\right]\right\} \\
\leq & l\left(1+\frac{1}{\Gamma(p+1)}\right)\left\{\bar{\mu}_{1} \sum_{j=1}^{m}\left|v_{j}\right| \frac{\sigma_{j}^{\zeta+\kappa}}{\Gamma(\zeta+\kappa+1)}\right.
\end{aligned}
$$




$$
\begin{aligned}
& \left.\quad+\bar{\mu}_{2}\left(\sum_{i=1}^{n}\left|\rho_{i}\right| \frac{\left(\eta_{i}^{\zeta+\kappa+1}-\xi_{i}^{\zeta+\kappa+1}\right)}{\Gamma(\zeta+\kappa+2)}+\frac{\left|a_{1}\right|}{\Gamma(\zeta+\kappa+1)}+\frac{\left|a_{2}\right|}{\Gamma(\zeta+\kappa)}\right)\right\}\|x-y\| \\
& +\chi\left[\bar{\mu}_{1} \sum_{j=1}^{m}\left|v_{j}\right| \frac{\sigma_{j}^{\zeta}}{\Gamma(\zeta+1)}+\bar{\mu}_{2}\left(\sum_{i=1}^{n}\left|\rho_{i}\right| \frac{\left(\eta_{i}^{\zeta+1}-\xi_{i}^{\zeta+1}\right)}{\Gamma(\zeta+2)}+\frac{\left|a_{1}\right|}{\Gamma(\zeta+1)}+\frac{\left|a_{2}\right|}{\Gamma(\zeta)}\right)\right] \\
& \quad \times\|x-y\| \\
& \leq \Lambda_{3}\|x-y\|,
\end{aligned}
$$

which, in view of condition (3.16), implies that $\mathcal{A}_{2}$ is a contraction.

(iii) $\mathcal{A}_{1}$ is compact and continuous. Continuity of $f$ implies that the operator $\mathcal{A}_{1}$ is continuous. Also, $\mathcal{A}_{1}$ is uniformly bounded on $B_{r}$ as

$$
\begin{aligned}
\left\|\mathcal{A}_{1} x\right\| & \leq \sup _{t \in[0,1]}\left\{\int_{0}^{t} \frac{(t-s)^{\zeta+\kappa-1}}{\Gamma(\zeta+\kappa)}\left|f\left(s, x(s), I^{p} x(s)\right)\right| d s+\chi \int_{0}^{t} \frac{(t-s)^{\zeta-1}}{\Gamma(\zeta)}|x(s)| d s\right\} \\
& \leq \frac{\|k\|}{\Gamma(\zeta+\kappa+1)}+\frac{\chi r}{\Gamma(\zeta+1)} .
\end{aligned}
$$

Next we show the compactness of the operator $\mathcal{A}_{1}$.

In view of $\left(C_{1}\right)$, we define $\sup _{(t, x, y) \in[0,1] \times B_{r} \times B_{r}}|f(t, x, y)|=\bar{f}$. Then, for $0<t_{1}<t_{2}<1$, we find that

$$
\begin{aligned}
\left|\mathcal{A}_{1} x\left(t_{2}\right)-\mathcal{A}_{1} x\left(t_{1}\right)\right| \\
\leq \mid \frac{1}{\Gamma(\zeta+\kappa)} \int_{0}^{t_{1}}\left(\left(t_{2}-s\right)^{\zeta+\kappa-1}-\left(t_{1}-s\right)^{\zeta+\kappa-1}\right) f\left(s, x(s), I^{p} x(s)\right) d s \\
\quad+\int_{t_{1}}^{t_{2}}\left(t_{2}-s\right)^{\zeta+\kappa-1} f\left(s, x(s), I^{p} x(s)\right) d s \\
\quad+\frac{\chi}{\Gamma(\zeta)} \int_{0}^{t_{1}}\left(\left(t_{2}-s\right)^{\zeta-1}-\left(t_{1}-s\right)^{\zeta-1}\right) x(s) d s+\int_{t_{1}}^{t_{2}}\left(t_{2}-s\right)^{\zeta-1} x(s) d s \mid \\
\leq \frac{\bar{f}}{\Gamma(\zeta+\kappa+1)}\left[2\left(t_{2}-t_{1}\right)^{\zeta+\kappa}+\left|t_{1}^{\zeta+\kappa}-t_{2}^{\zeta+\kappa}\right|\right] \\
\quad+\frac{\chi r}{\Gamma(\zeta+1)}\left[2\left(t_{2}-t_{1}\right)^{\zeta}+\left|t_{1}^{\zeta}-t_{2}^{\zeta}\right|\right] \rightarrow 0 \text { as } t_{2}-t_{1} \rightarrow 0,
\end{aligned}
$$

independently of $x \in B_{r}$. Thus, $\mathcal{A}_{1}$ is relatively compact on $B_{r}$. Hence, by the Arzela-Ascoli theorem, $\mathcal{A}_{1}$ is compact on $B_{r}$. In view of steps (i)-(iii), the hypothesis of Krasnoselskii's fixed point theorem [37] is satisfied. Thus, by the conclusion of Krasnoselskii's fixed point theorem, there exists a point $x \in B_{r}$ such that $x=\mathcal{A}_{1} x+\mathcal{A}_{2} x$, which corresponds to a solution of problem (1.1) on $[0,1]$. This completes the proof.

Theorem 3.3 Let $f:[0,1] \times \mathbb{R}^{2} \rightarrow \mathbb{R}$ be a continuous function, and let the following conditions hold:

$\left(C_{3}\right)$ There exist a function $p_{1} \in C\left([0,1], \mathbb{R}^{+}\right)$and a nondecreasing function $\psi: \mathbb{R}^{+} \rightarrow \mathbb{R}^{+}$ such that $\left|f\left(t, x, I^{p} x\right)\right| \leq p_{1}(t) \psi(|x|)$ for all $t \in[0,1], x \in \mathbb{R}$; 
$\left(C_{4}\right)$ There exists a positive constant $\delta$ such that

$$
\frac{\delta}{\left\|p_{1}\right\| \psi(\delta) \Lambda_{1}+\delta \Lambda_{2}}>1
$$

where $\Lambda_{1}$ and $\Lambda_{2}$ are respectively given by (3.14) and (3.15).

Then the boundary value problem (1.1) has at least one solution on $[0,1]$.

Proof We split the proof into several steps. In step 1, we show that the operator $\mathcal{A}: X \rightarrow X$ defined by (3.13) maps bounded sets into bounded sets in $X$. For a positive number $r$, let $B_{r}=\{x \in X:\|x\| \leq r\}$ be a bounded set in $X$. Then

$$
\begin{aligned}
& |(\mathcal{A} x)(t)| \\
& \leq \sup _{t \in[0,1]}\left\{\int_{0}^{t} \frac{(t-s)^{\zeta+\kappa-1}}{\Gamma(\zeta+\kappa)}\left|f\left(s, x(s), I^{p} x(s)\right)\right| d s+\chi \int_{0}^{t} \frac{(t-s)^{\zeta-1}}{\Gamma(\zeta)}|x(s)| d s\right. \\
& +\left|\mu_{1}(t)\right| \sum_{j=1}^{m}\left|v_{j}\right| \int_{0}^{\sigma_{j}}\left(\frac{\left(\sigma_{j}-s\right)^{\zeta+\kappa-1}}{\Gamma(\zeta+\kappa)}\left|f\left(s, x(s), I^{p} x(s)\right)\right|+\chi \frac{\left(\sigma_{j}-s\right)^{\zeta-1}}{\Gamma(\zeta)}|x(s)|\right) d s \\
& +\left|\mu_{2}(t)\right|\left[\sum _ { i = 1 } ^ { n } | \rho _ { i } | \int _ { \xi _ { i } } ^ { \eta _ { i } } \int _ { 0 } ^ { s } \left(\frac{(s-u)^{\zeta+\kappa-1}}{\Gamma(\zeta+\kappa)}\left|f\left(u, x(u), I^{p} x(u)\right)\right|\right.\right. \\
& \left.+\chi \frac{(s-u)^{\zeta-1}}{\Gamma(\zeta)}|x(u)|\right) d u d s \\
& +\left|a_{1}\right|\left(\int_{0}^{1} \frac{(1-s)^{\zeta+\kappa-1}}{\Gamma(\zeta+\kappa)}\left|f\left(s, x(s), I^{p} x(s)\right)\right| d s+\chi \int_{0}^{1} \frac{(1-s)^{\zeta-1}}{\Gamma(\zeta)}|x(s)| d s\right) \\
& \left.\left.+\left|a_{2}\right|\left(\int_{0}^{1} \frac{(1-s)^{\zeta+\kappa-2}}{\Gamma(\zeta+\kappa-1)}\left|f\left(s, x(s), I^{p} x(s)\right)\right| d s+\chi \int_{0}^{1} \frac{(1-s)^{\zeta-2}}{\Gamma(\zeta-1)}|x(s)| d s\right)\right]\right\} \\
& \leq\left\|p_{1}\right\| \psi(\|x\|)\left[\frac{1}{\Gamma(\zeta+\kappa+1)}+\bar{\mu}_{1} \sum_{j=1}^{m}\left|v_{j}\right| \frac{\sigma_{j}^{\zeta+\kappa}}{\Gamma(\zeta+\kappa+1)}\right. \\
& \left.+\bar{\mu}_{2}\left(\sum_{i=1}^{n}\left|\rho_{i}\right| \frac{\left(\eta_{i}^{\zeta+\kappa+1}-\xi_{i}^{\zeta+\kappa+1}\right)}{\Gamma(\zeta+\kappa+2)}+\frac{\left|a_{1}\right|}{\Gamma(\zeta+\kappa+1)}+\frac{\left|a_{2}\right|}{\Gamma(\zeta+\kappa)}\right)\right] \\
& +\chi\|x\|\left[\frac{1}{\Gamma(\zeta+1)}+\bar{\mu}_{1} \sum_{j=1}^{m}\left|v_{j}\right| \frac{\sigma_{j}^{\zeta}}{\Gamma(\zeta+1)}\right. \\
& \left.+\bar{\mu}_{2}\left(\sum_{i=1}^{n}\left|\rho_{i}\right| \frac{\left(\eta_{i}^{\zeta+1}-\xi_{i}^{\zeta+1}\right)}{\Gamma(\zeta+2)}+\frac{\left|a_{1}\right|}{\Gamma(\zeta+1)}+\frac{\left|a_{2}\right|}{\Gamma(\zeta)}\right)\right] \\
& \leq\left\|p_{1}\right\| \psi(\|x\|) \Lambda_{1}+\Lambda_{2}\|x\|,
\end{aligned}
$$

which, on taking the norm for $t \in[0,1]$, yields

$$
\|\mathcal{A} x\| \leq\left\|p_{1}\right\| \psi(r) \Lambda_{1}+\Lambda_{2} r
$$

Next we show that $\mathcal{A}$ maps bounded sets into equicontinuous sets of $X$. Let $\tau_{1}, \tau_{2} \in[0,1]$ with $\tau_{1}<\tau_{2}$ and $x \in B_{r}$. Then we obtain

$$
\left|\mathcal{A} x\left(\tau_{2}\right)-\mathcal{A} x\left(\tau_{1}\right)\right|
$$




$$
\begin{aligned}
\leq & \frac{\left\|p_{1}\right\| \psi(r)}{\Gamma(\zeta+\kappa+1)}\left[2\left(\tau_{2}-\tau_{1}\right)^{\zeta+\kappa}+\left|\tau_{1}^{\zeta+\kappa}-\tau_{2}^{\zeta+\kappa}\right|\right]+\frac{\chi r}{\Gamma(\zeta+1)}\left[2\left(\tau_{2}-\tau_{1}\right)^{\zeta}+\left|\tau_{1}^{\zeta}-\tau_{2}^{\zeta}\right|\right] \\
& +\frac{\left|B_{2}\right|\left|\tau_{2}^{\zeta+1}-\tau_{1}^{\zeta+1}\right|}{|\Delta| \Gamma(\zeta+2)} \sum_{j=1}^{m}\left|\nu_{j}\right|\left[\frac{\left\|p_{1}\right\| \psi(r) \sigma_{j}^{\zeta+\kappa}}{\Gamma(\zeta+\kappa+1)}+\frac{\chi r \sigma_{j}^{\zeta}}{\Gamma(\zeta+1)}\right] \\
& +\frac{\left|A_{2} \| \tau_{2}^{\zeta+1}-\tau_{1}^{\zeta+1}\right|}{|\Delta| \Gamma(\zeta+2)}\left[\sum_{i=1}^{n}\left|\rho_{i}\right|\left(\frac{\left\|p_{1}\right\| \psi(r)\left(\eta_{i}^{\zeta+\kappa+1}-\xi_{i}^{\zeta+\kappa+1}\right)}{\Gamma(\zeta+\kappa+2)}+\frac{\chi r\left(\eta_{i}^{\zeta+1}-\xi_{i}^{\zeta+1}\right)}{\Gamma(\zeta+2)}\right)\right. \\
& \left.+\left|a_{1}\right|\left(\frac{\left\|p_{1}\right\| \psi(r)}{\Gamma(\zeta+\kappa+1)}+\frac{\chi r}{\Gamma(\zeta+1)}\right)+\left|a_{2}\right|\left(\frac{\left\|p_{1}\right\| \psi(r)}{\Gamma(\zeta+\kappa)}+\frac{\chi r}{\Gamma(\zeta)}\right)\right] \rightarrow 0
\end{aligned}
$$

as $\tau_{2}-\tau_{1} \rightarrow 0$ independently of $x \in B_{r}$. In view of the foregoing arguments, we deduce by the Arzela-Ascoli theorem that the operator $\mathcal{A}: X \rightarrow X$ is completely continuous.

Lastly, we establish that the set of all solutions $x$ to equation $x=\theta \mathcal{A} x$ is bounded for $\theta \in[0,1]$. As argued in proving that the operator $\mathcal{A}$ is bounded, one can obtain that

$$
\frac{\|x\|}{\left\|p_{1}\right\| \psi(\|x\|) \Lambda_{1}+\Lambda_{2}\|x\|} \leq 1
$$

On the other hand, we can find a positive number $\delta$ such that $\|x\| \neq \delta$ by assumption $\left(C_{4}\right)$. Let us consider a set $V=\{x \in X:\|x\|<\delta\}$. Notice that the operator $\mathcal{A}: \bar{V} \rightarrow X$ is continuous and completely continuous. From the definition of $V$, we cannot find any $x \in$ $\partial V$ satisfying the equation $x=\theta \mathcal{A}(x)$ for some $\theta \in(0,1)$. Thus we conclude by the LeraySchauder nonlinear alternative [38] that there exists a fixed point $x \in \bar{V}$ for the operator $\mathcal{A}$, which is a solution of problem (1.1). This completes the proof.

\subsection{Uniqueness result}

Theorem 3.4 Assume that $f:[0,1] \times \mathbb{R}^{2} \rightarrow \mathbb{R}$ is a continuous function satisfying condition $\left(C_{1}\right)$. Then there exists a unique solution for the boundary value problem $(1.1)$ on $[0,1]$ provided that

$$
l\left(1+\frac{1}{\Gamma(p+1)}\right) \Lambda_{1}+\Lambda_{2}<1
$$

where $\Lambda_{1}$ and $\Lambda_{2}$ are respectively given by (3.14) and (3.15).

Proof Consider a closed ball $B_{\bar{r}}=\{x \in X:\|x\| \leq \bar{r}\}$, where

$$
\bar{r} \geq \frac{M_{0} \Lambda_{1}}{1-\left(l\left(1+\frac{1}{\Gamma(p+1)}\right) \Lambda_{1}+\Lambda_{2}\right)}, \quad M_{0}=\sup _{t \in[0,1]}|f(t, 0,0)|<\infty,
$$

and show that $\mathcal{A} B_{\bar{r}} \subset B_{\bar{r}}(\mathcal{A}: X \rightarrow X$ is defined by (3.13)).

Letting $\widehat{f}_{x}(s)=f\left(s, x(s), I^{p} x(s)\right), x \in B_{\bar{r}}$ and using condition $\left(C_{1}\right)$, we obtain

$$
\begin{aligned}
\left|\widehat{f}_{x}(s)\right| & =\left|f\left(s, x(s), I^{p} x(s)\right)-f(s, 0,0)+f(s, 0,0)\right| \\
& \leq\left|f\left(s, x(s), I^{p} x(s)\right)-f(s, 0,0)\right|+|f(s, 0,0)|
\end{aligned}
$$




$$
\begin{aligned}
& \leq l\left(|x(s)|+\left|I^{p} x(s)\right|\right)+|f(s, 0,0)| \\
& \leq l\left(1+\frac{1}{\Gamma(p+1)}\right)\|x\|+M_{0} \leq l\left(1+\frac{1}{\Gamma(p+1)}\right) \bar{r}+M_{0} .
\end{aligned}
$$

Then

$$
\begin{aligned}
|\mathcal{A} x(t)| \leq & \sup _{t \in[0,1]}\left\{\int_{0}^{t} \frac{(t-s)^{\zeta+\kappa-1}}{\Gamma(\zeta+\kappa)}\left|\widehat{f}_{x}(s)\right| d s+\chi \int_{0}^{t} \frac{(t-s)^{\zeta-1}}{\Gamma(\zeta)}|x(s)| d s\right. \\
& +\left|\mu_{1}(t)\right| \sum_{j=1}^{m}\left|v_{j}\right| \int_{0}^{\sigma_{j}}\left(\frac{\left(\sigma_{j}-s\right)^{\zeta+\kappa-1}}{\Gamma(\zeta+\kappa)}\left|\widehat{f}_{x}(s)\right|+\chi \frac{\left(\sigma_{j}-s\right)^{\zeta-1}}{\Gamma(\zeta)}|x(s)|\right) d s \\
& +\left|\mu_{2}(t)\right|\left[\sum_{i=1}^{n}\left|\rho_{i}\right| \int_{\xi_{i}}^{\eta_{i}} \int_{0}^{s}\left(\frac{(s-u)^{\zeta+\kappa-1}}{\Gamma(\zeta+\kappa)}\left|\widehat{f}_{x}(s)\right|+\chi \frac{(s-u)^{\zeta-1}}{\Gamma(\zeta)}|x(u)|\right) d u d s\right. \\
& +\left|a_{1}\right|\left(\int_{0}^{1} \frac{(1-s)^{\zeta+\kappa-1}}{\Gamma(\zeta+\kappa)}\left|\widehat{f}_{x}(s)\right| d s+\chi \int_{0}^{1} \frac{(1-s)^{\zeta-1}}{\Gamma(\zeta)}|x(s)| d s\right) \\
& \left.\left.+\left|a_{2}\right|\left(\int_{0}^{1} \frac{(1-s)^{\zeta+\kappa-2}}{\Gamma(\zeta+\kappa-1)}\left|\widehat{f}_{x}(s)\right| d s+\chi \int_{0}^{1} \frac{(1-s)^{\zeta-2}}{\Gamma(\zeta-1)}|x(s)| d s\right)\right]\right\} \\
\leq & \left(l\left(1+\frac{1}{\Gamma(p+1)}\right) \Lambda_{1}+\Lambda_{2}\right) \bar{r}+M_{0} \Lambda_{1} \leq \bar{r},
\end{aligned}
$$

which implies that $\|\mathcal{A} x\| \leq \bar{r}$. Thus $\mathcal{A} B_{\bar{r}} \subset B_{\bar{r}}$.

Now, for $x, y \in X$ and for each $t \in[0,1]$, we obtain

$$
\begin{aligned}
\| \mathcal{A} x & -\mathcal{A} y \| \\
\leq & \sup _{t \in[0,1]}\left\{\int_{0}^{t} \frac{(t-s)^{\zeta+\kappa-1}}{\Gamma(\zeta+\kappa)}\left|\widehat{f}_{x}(s)-\widehat{f}_{y}(s)\right| d s+\chi \int_{0}^{t} \frac{(t-s)^{\zeta-1}}{\Gamma(\zeta)}|x(s)-y(s)| d s\right. \\
& +\left|\mu_{1}(t)\right| \sum_{j=1}^{m}\left|v_{j}\right| \int_{0}^{\sigma_{j}}\left(\frac{\left(\sigma_{j}-s\right)^{\zeta+\kappa-1}}{\Gamma(\zeta+\kappa)}\left|\widehat{f}_{x}(s)-\widehat{f}_{y}(s)\right|+\chi \frac{\left(\sigma_{j}-s\right)^{\zeta-1}}{\Gamma(\zeta)}|x(s)-y(s)|\right) d s \\
& +\left|\mu_{2}(t)\right|\left[\sum _ { i = 1 } ^ { n } | \rho _ { i } | \int _ { \xi _ { i } } ^ { \eta _ { i } } \int _ { 0 } ^ { s } \left(\frac{(s-u)^{\zeta+\kappa-1}}{\Gamma(\zeta+\kappa)}\left|\widehat{f}_{x}(u)-\widehat{f}_{y}(u)\right|\right.\right. \\
& \left.+\chi \frac{(s-u)^{\zeta-1}}{\Gamma(\zeta)}|x(u)-y(u)|\right) d u d s \\
& +\left|a_{1}\right|\left(\int_{0}^{1} \frac{(1-s)^{\zeta+\kappa-1}}{\Gamma(\zeta+\kappa)}\left|\widehat{f}_{x}(s)-\widehat{f}_{y}(s)\right| d s+\chi \int_{0}^{1} \frac{(1-s)^{\zeta-1}}{\Gamma(\zeta)}|x(s)-y(s)| d s\right) \\
& \left.\left.+\left|a_{2}\right|\left(\int_{0}^{1} \frac{(1-s)^{\zeta+\kappa-2}}{\Gamma(\zeta+\kappa-1)}\left|\widehat{f}_{x}(s)-\widehat{f}_{y}(s)\right| d s+\chi \int_{0}^{1} \frac{(1-s)^{\zeta-2}}{\Gamma(\zeta-1)}|x(s)-y(s)| d s\right)\right]\right\} \\
\leq & l\left(1+\frac{1}{\Gamma(p+1)}\right)\left\{\frac{1}{\Gamma(\zeta+\kappa+1)}+\bar{\mu}_{1} \sum_{j=1}^{m}\left|v_{j}\right| \frac{\sigma_{j}^{\zeta+\kappa}}{\Gamma(\zeta+\kappa+1)}\right. \\
& \left.+\bar{\mu}_{2}\left(\sum_{i=1}^{n}\left|\rho_{i}\right| \frac{\left(\eta_{i}^{\zeta+\kappa+1}-\xi_{i}^{\zeta+\kappa+1}\right)}{\Gamma(\zeta+\kappa+2)}+\frac{\left|a_{1}\right|}{\Gamma(\zeta+\kappa+1)}+\frac{\left|a_{2}\right|}{\Gamma(\zeta+\kappa)}\right)\right\}\|x-y\|
\end{aligned}
$$




$$
\begin{aligned}
& \quad+\chi\left[\frac{1}{\Gamma(\zeta+1)}+\bar{\mu}_{1} \sum_{j=1}^{m}\left|v_{j}\right| \frac{\sigma_{j}^{\zeta}}{\Gamma(\zeta+1)}\right. \\
& \left.+\bar{\mu}_{2}\left(\sum_{i=1}^{n}\left|\rho_{i}\right| \frac{\left(\eta_{i}^{\zeta+1}-\xi_{i}^{\zeta+1}\right)}{\Gamma(\zeta+2)}+\frac{\left|a_{1}\right|}{\Gamma(\zeta+1)}+\frac{\left|a_{2}\right|}{\Gamma(\zeta)}\right)\right]\|x-y\| \\
& \leq\left[l\left(1+\frac{1}{\Gamma(p+1)}\right) \Lambda_{1}+\Lambda_{2}\right]\|x-y\|,
\end{aligned}
$$

which shows that $\mathcal{A}$ is a contraction in view of condition (3.17). Hence we deduce by the Banach contraction mapping principle that the operator $\mathcal{A}$ has a unique fixed point, which corresponds to a unique solution of problem $(1.1)$ on $[0,1]$. This completes the proof.

\section{Examples}

Consider the following Langevin equation with fixed fractional orders:

$$
{ }^{c} D^{\frac{3}{2}}\left({ }^{c} D^{\frac{1}{2}}+\frac{1}{4}\right) x(t)=f\left(t, x(t), I^{\frac{3}{4}} x(t)\right), \quad t \in[0,1],
$$

subject to the multi-point and multi-strip boundary conditions

$$
x(0)=\sum_{j=1}^{m} v_{j} x\left(\sigma_{j}\right), \quad x^{\prime}(0)=0, \quad a_{1} x(1)+a_{2} x^{\prime}(1)=\sum_{i=1}^{n} \rho_{i} \int_{\xi_{i}}^{\eta_{i}} x(s) d s
$$

where $\zeta=\frac{1}{2}, \kappa=\frac{3}{2}, p=\frac{3}{4}, \chi=\frac{1}{4}, m=3, n=3, a_{1}=1, a_{2}=1, \sigma_{1}=\frac{1}{28}, \sigma_{2}=\frac{1}{21}, \sigma_{3}=\frac{1}{14}$, $\xi_{1}=\frac{1}{9}, \xi_{2}=\frac{3}{9}, \xi_{3}=\frac{5}{9}, \eta_{1}=\frac{2}{9}, \eta_{2}=\frac{4}{9}, \eta_{3}=\frac{6}{9}, v_{1}=\frac{1}{15}, v_{2}=\frac{1}{10}, v_{3}=\frac{1}{5}, \rho_{1}=\frac{1}{12}, \rho_{2}=\frac{1}{6}, \rho_{3}=\frac{1}{4}$, and $f\left(t, x, I^{p} x\right)$ will be fixed later. Using the given values, we find that $\Lambda_{1} \approx 1.111762, \Lambda_{2} \approx$ $0.511583\left(\Lambda_{1}\right.$ and $\Lambda_{2}$ are respectively given by (3.14) and (3.15)).

In order to illustrate Theorem 3.2, we take the following nonlinear function in (4.1):

$$
f\left(t, x, I^{\frac{3}{4}} x\right)=\frac{1}{\sqrt{t^{2}+144}}\left(\tan ^{-1} x+e^{-t} \int_{0}^{t} \frac{(t-s)^{\frac{-1}{4}}}{\Gamma\left(\frac{3}{4}\right)} x d s+\frac{1}{3}\right), \quad 0<t<1,
$$

which obviously satisfies the Lipschitz condition $\left(C_{1}\right)$ with $l=1 / 12$, that is,

$$
\left|f\left(t, x, I^{\frac{3}{4}} x\right)-f\left(t, y, I^{\frac{3}{4}} y\right)\right| \leq \frac{1}{12}\left(1+\frac{1}{\Gamma(7 / 4)}\right)|x-y| .
$$

Moreover, $\Lambda_{3} \approx 0.335938<1$. Clearly the hypothesis of Theorem 3.2 holds true, and consequently its conclusion applies to problem (4.1)-(4.2) with $f\left(t, x, I^{\frac{3}{4}} x\right)$ given by (4.3).

To demonstrate the application of Theorem 3.3, we consider

$$
f\left(t, x, I^{\frac{3}{4}} x\right)=\frac{1}{\sqrt{25+t^{2}}}\left(\sin x+\frac{|x|}{4(1+|x|)}+I^{\frac{3}{4}} x+\frac{1}{4}\right), \quad t \in[0,1]
$$

in (4.1) and note that

$$
\left|f\left(t, x, I^{\frac{3}{4}} x\right)\right| \leq \frac{1}{\sqrt{25+t^{2}}}\left(\frac{1}{2}+\left(1+\frac{1}{\Gamma\left(\frac{7}{4}\right)}\right)\|x\|\right),
$$


with $p_{1}(t)=\frac{1}{\sqrt{25+t^{2}}},\left\|p_{1}\right\|=\frac{1}{5}$, and $\psi(\|x\|)=\frac{1}{2}+\left(1+\frac{1}{\Gamma\left(\frac{7}{4}\right)}\right)\|x\|$. By condition $\left(C_{4}\right)$, we find that $M>4.60723755$. Thus all the assumptions of Theorem 3.3 are satisfied. Hence, there exists at least one solution for problem (4.1)-(4.2) with $f\left(t, x, I^{\frac{3}{4}} x\right)$ given by $(4.4)$ on $[0,1]$. Since $l\left(1+\frac{1}{\Gamma(7 / 4)}\right) \Lambda_{1}+\Lambda_{2} \approx 0.705036<1$, therefore problem $(4.1)-(4.2)$ with $f\left(t, x, I^{\frac{3}{4}} x\right)$ given by (4.3) has a unique solution on $[0,1]$ by Theorem 3.4 .

\section{Conclusions}

We have discussed the solvability of a nonlinear Langevin equation involving Caputo fractional derivatives of different orders with nonlinearity depending upon the unknown function together with its Riemann-Liouville fractional integral, subject to nonlocal multipoint and multi-strip boundary conditions. The results obtained in this paper are new and strengthen the literature on Langevin equation. Moreover, several results follow as special cases of the ones presented in this paper by fixing the parameters in the boundary conditions. For example, our results correspond to the boundary conditions:

- $x(0)=0, x^{\prime}(0)=0, a_{1} x(1)+a_{2} x^{\prime}(1)=0$ if we take all $\rho_{i}=0=v_{j}, i=1,2, \ldots, n$, $j=1,2, \ldots, m$, and $a_{1} \neq 0 \neq a_{2}$;

- $x(0)=\sum_{j=1}^{m} v_{j} x\left(\sigma_{j}\right), x^{\prime}(0)=0, a_{1} x(1)+a_{2} x^{\prime}(1)=0$ by fixing all $\rho_{i}=0, i=1,2, \ldots, n$;

- $x(0)=0, x^{\prime}(0)=0, a_{1} x(1)+a_{2} x^{\prime}(1)=\sum_{i=1}^{n} \rho_{i} \int_{\xi_{i}}^{\eta_{i}} x(s) d s$ by taking all $v_{j}=0$, $j=1,2, \ldots, m$;

- $x(0)=\sum_{j=1}^{m} v_{j} x\left(\sigma_{j}\right), x^{\prime}(0)=0, x(1)=\sum_{i=1}^{n} \rho_{i} \int_{\xi_{i}}^{\eta_{i}} x(s) d s$ for $a_{1}=1, a_{2}=0$;

- $x(0)=\sum_{j=1}^{m} v_{j} x\left(\sigma_{j}\right), x^{\prime}(0)=0, \sum_{i=1}^{n} \rho_{i} \int_{\xi_{i}}^{\eta_{i}} x(s) d s=0$ when $a_{1}=0=a_{2}$ and all $\rho_{i}$ $(i=1,2, \ldots, n)$ and $v_{j}(j=1,2, \ldots, m)$ are not zero.

\section{Acknowledgements}

This project was funded by the Deanship of Scientific Research (DSR), King Abdulaziz University, Jeddah, Saudi Arabia under grant no. (KEP-MSc-10-130-39). The authors, therefore, acknowledge with thanks DSR technical and financial support. The authors also appreciate the reviewers for their useful comments on our paper.

\section{Funding}

This project was funded by the Deanship of Scientific Research (DSR), King Abdulaziz University, Jeddah, Saudi Arabia under grant no. (KEP-MSc-10-130-39).

\section{Abbreviations}

Not applicable.

Availability of data and materials

Not applicable.

\section{Competing interests}

The authors declare that they have no competing interests.

\section{Authors' contributions}

Each of the authors, BA, AA, and SS, contributed equally to each part of this work. All authors read and approved the final manuscript.

\section{Publisher's Note}

Springer Nature remains neutral with regard to jurisdictional claims in published maps and institutional affiliations.

Received: 3 January 2019 Accepted: 30 January 2019 Published online: 12 February 2019

\section{References}

1. Langevin, P.: Sur la théorie du mouvement brownien. C. R. Acad. Sci. Paris 146, 530-533 (1908) (in French)

2. Klages, R., Radons, G., Sokolov, I.M.: Anomalous Transport: Foundations and Applications. Wiley, Weinheim (2008)

3. Kubo, R.: The fluctuation-dissipation theorem. Rep. Prog. Phys. 29, 255-284 (1966)

4. Kubo, R., Toda, M., Hashitsume, N.: Statistical Physics II, 2nd edn. Springer, Berlin (1991)

5. Magin, R.L.: Fractional Calculus in Bioengineering. Begell House, Chicago (2006)

6. Kilbas, A.A., Srivastava, H.M., Trujillo, J.J.: Theory and Applications of Fractional Differential Equations. North-Holland Mathematics Studies, vol. 204. Elsevier, Amsterdam (2006) 
7. Sabatier, J., Agrawal, O.P., Machado, J.A.T.: Advances in Fractional Calculus: Theoretical Developments and Applications in Physics and Engineering. Springer, Dordrecht (2007)

8. Mainardi, F.: Fractional Calculus and Waves in Linear Viscoelasticity: An Introduction to Mathematical Models. Imperial College Press, Singapore (2010)

9. Fallahgoul, H.A., Focardi, S.M., Fabozzi, F.J.: Fractional Calculus and Fractional Processes with Applications to Financial Economics: Theory and Application. Elsevier, London (2017)

10. Carvalho, A., Pinto, C.M.A.: A delay fractional order model for the co-infection of malaria and HIV/AIDS. Int. J. Dyn. Control 5, 168-186 (2017)

11. Ahmad, B., Sivasundaram, S.: On four-point nonlocal boundary value problems of nonlinear integro-differential equations of fractional order. Appl. Math. Comput. 217, 480-487 (2010)

12. Zhai, C., Xu, L.: Properties of positive solutions to a class of four-point boundary value problem of Caputo fractional differential equations with a parameter. Commun. Nonlinear Sci. Numer. Simul. 19, 2820-2827 (2014)

13. Li, B., Sun, S., Li, Y., Zhao, P.: Multi-point boundary value problems for a class of Riemann-Liouville fractional differential equations. Adv. Differ. Equ. 2014, 151 (2014)

14. Zhang, L., Ahmad, B., Wang, G.: Successive iterations for positive extremal solutions of nonlinear fractional differential equations on a half line. Bull. Aust. Math. Soc. 91, 116-128 (2015)

15. Henderson, J., Luca, R.: Nonexistence of positive solutions for a system of coupled fractional boundary value problems. Bound. Value Probl. 2015, 138 (2015)

16. Ntouyas, S.K., Etemad, S.: On the existence of solutions for fractional differential inclusions with sum and integral boundary conditions. Appl. Math. Comput. 266, 235-243 (2015)

17. Qarout, D., Ahmad, B., Alsaedi, A.: Existence theorems for semilinear Caputo fractional differential equations with nonlocal discrete and integral boundary conditions. Fract. Calc. Appl. Anal. 19, 463-479 (2016)

18. Zhou, Y., Ahmad, B., Alsaedi, A.: Existence of nonoscillatory solutions for fractional neutral differential equations. Appl. Math. Lett. 72, 70-74 (2017)

19. Chang, Y.-K., Pereira, A., Ponce, R.: Approximate controllability for fractional differential equations of Sobolev type via properties on resolvent operators. Fract. Calc. Appl. Anal. 20,963-987 (2017)

20. Wang, G., Pei, K., Agarwal, R.P., Zhang, L., Ahmad, B.: Nonlocal Hadamard fractional boundary value problem with Hadamard integral and discrete boundary conditions on a half-line. J. Comput. Appl. Math. 343, 230-239 (2018)

21. Zhou, Y.: Attractivity for fractional differential equations in Banach space. Appl. Math. Lett. 75, 1-6 (2018)

22. Tariboon, J., Cuntavepanit, A., Ntouyas, S.K., Nithiarayaphaks, W.: Separated boundary value problems of sequential Caputo and Hadamard fractional differential equations. J. Funct. Spaces 2018, Article ID 6974046 (2018)

23. Ahmad, B., Luca, R.: Existence of solutions for sequential fractional integro-differential equations and inclusions with nonlocal boundary conditions. Appl. Math. Comput. 339, 516-534 (2018)

24. Eab, C.H., Lim, S.C.: Fractional generalized Langevin equation approach to single-file diffusion. Physica A 389, 2510-2521 (2010)

25. Eule, S., Friedrich, R., Jenko, F., Kleinhans, D.: Langevin approach to fractional diffusion equations including inertial effects. J. Phys. Chem. B 111(39), 11474-11477 (2007)

26. West, B.J., Latka, M.: Fractional Langevin model of gait variability. J. NeuroEng. Rehabil. 2, 24 (2005)

27. Lim, S.C., Li, M., Teo, L.P.: Langevin equation with two fractional orders. Phys. Lett. A 372, 6309-6320 (2008)

28. Ahmad, B., Nieto, J., Alsaedi, A., El-Shahed, M.: A study of nonlinear Langevin equation involving two fractional orders in different intervals. Nonlinear Anal., Real World Appl. 13, 599-606 (2012)

29. Ahmad, B., Ntouyas, S.K.: New existence results for differential inclusions involving Langevin equation with two indices. J. Nonlinear Convex Anal. 14(3), 437-450 (2013)

30. Torres, C.: Existence of solution for fractional Langevin equation: variational approach. Electron. J. Qual. Theory Differ. Equ. 2014, Article ID 54 (2014)

31. Sudsutad, W., Ahmad, B., Ntouyas, S.K., Tariboon, J.: Impulsively hybrid fractional quantum Langevin equation with boundary conditions involving Caputo $q_{k}$-fractional derivatives. Chaos Solitons Fractals 91, 47-62 (2016)

32. Baghani, O.: On fractional Langevin equation involving two fractional orders. Commun. Nonlinear Sci. Numer. Simul. 42,675-681 (2017)

33. Li, B., Sun, S., Sun, Y.: Existence of solutions for fractional Langevin equation with infinite-point boundary conditions. J. Appl. Math. Comput. 53, 683-692 (2017)

34. Muensawat, T., Ntouyas, S.K., Tariboon, J.: Systems of generalized Sturm-Liouville and Langevin fractional differential equations. Adv. Differ. Equ. 2017, Article ID 63 (2017)

35. Fazli, H., Nieto, J.J.: Fractional Langevin equation with anti-periodic boundary conditions. Chaos Solitons Fractals 114 , 332-337 (2018)

36. Zhou, Z., Qiao, Y.: Solutions for a class of fractional Langevin equations with integral and anti-periodic boundary conditions. Bound. Value Probl. 2018, Article ID 152 (2018)

37. Krasnoselskii, M.A.: Two remarks on the method of successive approximations. Usp. Mat. Nauk 10, $123-127$ (1955)

38. Granas, A., Dugundji, J.: Fixed Point Theory. Springer, New York (2003) 